\title{
Superconductivity with $s$ and $p$ symmetries in an extended Hubbard model with correlated hopping *
}

\author{
A.A.Aligia ${ }^{1}$, E. Gagliano ${ }^{1}$, Liliana Arrachea ${ }^{2}$ and K. Hallberg ${ }^{1}$ \\ 1 Centro Atómico Bariloche and Instituto Balseiro, Comisión Nacional de Energía Atómica, 8400 Bariloche, Argentina \\ ${ }^{2}$ Max-Planck-Institut für Physik komplexer Systeme, Nöthnitzer Str. 38, 01187 Dresden, Germany
}

We consider a generalized Hubbard model with on-site and nearest-neighbour repulsions $U$ and $V$ respectively, and nearest-neighbour hopping for spin up (down) which depends on the total occupation $n_{b}$ of spin down (up) electrons on both sites involved. The hopping parameters are $t_{A A}, t_{A B}$ and $t_{B B}$ for $n_{b}=0,1,2$ respectively. We briefly summarize results which support that the model exhibits $s$-wave superconductivity for certain parameters and extend them by studying the Berry phases. Using a generalized Hartree-Fock(HF) BCS decoupling of the two and three-body terms, we obtain that at half filling, for $t_{A B}<t_{A A}=t_{B B}$ and sufficiently small $U$ and $V$ the model leads to triplet $p$-wave superconductivity for a simple cubic lattice in any dimension. In one dimension, the resulting phase diagram is compared with that obtained numerically using two quantized Berry phases (topological numbers) as order parameters. While this novel method supports the previous results, there are quantitative differences.

\section{INTRODUCTION}

Since the discovery of high $T_{c}$ much effort has been devoted to the study of the pairing mechanisms in highly correlated electronic systems. Two very well studied microscopic models for such systems are the Hubbard and $t-J$ models. While the search for superconductivity in the positive $J$ Hubbard model by numerical methods has failed so fart $t$, $t-J$-like models exhibit $d$-wave superconductivity for certain parameters 610.

Although most of the high $T_{c}$ superconducting materials have a two-dimensional character, calculations in one dimension (1D) have been very useful. In one dimension the Hubbard and supersymmetric $(J=2 t) t-J$ models can be exactly solved using the Bethe ansatz11, 22 . It has been shown that for this particular case of the $t-J$ model, the ground state consists of bound states with a gapless excitation spectrum, but for $J<2 t$ bound states exist for large enough densities 13 .

Other strongly correlated models that introduce correlated hopping interactions are subject of current research [14-51]. In 1989, Hirsch 14 proposed a model for

\footnotetext{
${ }^{*}$ Dedicated to Prof. Dr. J. Zittartz on the occasion of his $60^{\text {th }}$ birthday
}

the description of oxide superconductors by considering the holes in a nearly filled band as charge carriers. The Hamiltonian in standard notation reads:

$$
\begin{array}{r}
H_{H}=-\sum_{j=1}^{L} \sum_{\sigma= \pm 1}\left(c_{j, \sigma}^{\dagger} c_{j+1, \sigma}+c_{j+1, \sigma}^{\dagger} c_{j, \sigma}\right) \times \\
\left(1-\frac{\Delta t}{2}\left(n_{j,-\sigma}+n_{j+1,-\sigma}\right)\right)
\end{array}
$$

In contrast to the Hubbard model, the hopping amplitude for single-particle hopping to a nearest-neighbour site depends on the occupancy of one of the sites involved in the process.

A few years ago, a new integrable model of strongly correlated fermionic systems for hole superconductivity has been introduced 15 . It 6 . It is a modified version of Hirsch's model (11) and its Hamiltonian reads:

$$
\begin{array}{r}
H_{B}=-\sum_{j=1}^{L} \sum_{\sigma= \pm 1}\left(c_{j, \sigma}^{\dagger} c_{j+1, \sigma}+c_{j+1, \sigma}^{\dagger} c_{j, \sigma}\right) \times \\
\left(1-\Delta t n_{j+(1+\sigma) / 2,-\sigma}\right)
\end{array}
$$

In $1 \mathrm{D}$, it has been solved using the Bethe ansatz in three parameter regimes: $0<\Delta t<1, \Delta t<01517$ and $\Delta t>1$ 18. The two first regimes are related by a particlehole transformation and present similar behaviour to the Hirsch model (1): there is a good quantitative agreement between the ground state energies, both models show gapless charge excitations and spin excitations with a finite gap for all densities and also dominating superconducting correlations for densities larger than a critical value 19 . Instead, for the case with $\Delta t>1$, both models (1) and 2) behave quite differently: while the spin gap in the Hirsch model vanishes and there are no indications of superconductivity, the gap remains finite and superconducting correlations are present for small doping in the Bariev model (2).

An important interaction that has not been considered in the above models is the Coulomb repulsion. We consider a more complete Hamiltonian that includes onsite $U$ and nearest-neighbour $V$ interactions between the particles, and contains a more general kinetic term with different hopping parameters, depending on the total occupation of the sites involved:

$$
\begin{aligned}
H= & -\sum_{<i j>\sigma} \Phi_{i j}\left(c_{i,-\sigma}^{\dagger} c_{j,-\sigma}+h c\right)+U \sum_{i} n_{i \uparrow} n_{i \downarrow}+ \\
& V \sum_{<i j>} n_{i} n_{j}
\end{aligned}
$$




$$
\begin{gathered}
\Phi_{i j}=t_{A A}\left(1-n_{i \sigma}\right)\left(1-n_{j \sigma}\right)+t_{B B} n_{i \sigma} n_{j \sigma}+ \\
t_{A B}\left(n_{i \sigma}+n_{j \sigma}-2 n_{i \sigma} n_{j \sigma}\right)
\end{gathered}
$$

The model (11) corresponds to taking $t_{A A}+t_{B B}-$ $2 t_{A B}=0, U=V=0$ and $\Delta t / 2=1-t_{A B} / t_{A A}$, and the Hubbard model is obtained when $V=0, t_{A A}=$ $t_{B B}=t_{A B}$. The case $V=0$ has been derived as the model that describes the low energy excitations of intermediate-valence systems 20 and "hole" and cuprate superconductors2122. The medel with $V$ was obtained for cuprate superconductors 2324 and proposed to describe the benzene molecule25. 26 .

For $t_{A B}=V=0$ and $\left|t_{A A}\right|=\left|t_{B B}\right|$ an exact solution of (3) exists in 1D27 30, but this case is too peculiar, as discussed later. Hamiltonian (3) supplemented by a hopping of pairs of the type $t_{p} \sum_{\langle i j\rangle} c_{i, \uparrow}^{\dagger} c_{i, \uparrow}^{\dagger} c_{j} \uparrow c_{j, \downarrow}$ has also been solved in 1D using Bethe ansatz 133 for $U=$ $-2 t_{p}$ and $t_{A A} t_{B B}=t_{A B}^{2}$. Its behaviour is similar to that of the Hubbard model. For negative $U$ the model displays superconductivity. A more general model including this one and that of Bariev for particylar parameters has also been solved using Bethe ansatz 34 .

For $t_{A B}=0,\left|t_{A A}\right|=\left|t_{B B}\right|=t, U, V \neq 0$ the phase diagram of the model (3) has been investigated previously 20.35 , 37 and some exact results have been found for the half-filled system (density of particles $n=1$ ): a) in any arbitrary lattice in $D$ dimensions with coordination number $z=2 D$ the ground state is a Mott insulator (MI) with all sites singly occupied if $U>z$ $\left.\max \left(V,\left|t_{A A}\right|+\left|t_{B B}\right|\right) ; \mathrm{b}\right)$ the ground state is a charge density wave (CDW) if $V>\max \left(U / z, U / 2+\left|t_{A A}\right|+\left|t_{B B}\right|\right) / 2$ (simple cubic lattices) ; c) there is a region with mobile carriers which we call metallic (M) between these two phases, the M-MI boundary being at $U_{M-M I}=$ $z\left(\left|t_{A A}\right| t_{\beta} B \mid\right)$ (for $D=1$ this state is a "non-conducting metal" 30.36); d) for $D=1$ the M-CDW boundary is at $V_{M-C D W}=\left(U / 2+\left|t_{A A}\right|+\left|t_{B B}\right|\right) / 2$.

For $0<t_{A B}<t_{A A}=t_{B B}=t, U, V \neq 0$ the phase diagram at $n=1$ has been studied numerically in $1 \mathrm{D}$ and $2 \mathrm{D}$ and within mean-field approximation 3739 . The high spin degeneracy of the MI phase for $t_{A B}=0$ is lifted and gives place to a spin density wave (SDW) phase. There exists a metallic phase (the detailed nature of which will be discussed later) for small values of $U$ and $V$, which shrinks as $t_{A B} \rightarrow t$. When $t_{A B}=t$ (Hubbard limit), several results indicate that there is no $M$ phase, except, eventually, on the second-order transition line between the CDW and SDW phases, that ends at a tricritical point. The position of this point is, to date, matwell determined and should be around $U \sim 2 V \sim 4 t 5256$.

The aim of the present work is to study, by numerical and analytical methods, possible existence of superconductivity in the model (3) in regions of parameters for which exact results are not available. Special attention is paid to the occurrence of exotic $p$-wave triplet superconductivity (TS). In particular, we show strong evidence that the extension to finite $t_{A B}$ of the above mentioned
M phase displays TS. Some evidence of triplet superconductivity exists in the extended Hubbard model (Eq. 3 with $t_{A A} \overline{t_{A B}}=t_{B B}$ ) very near the line $U=-2 \mathrm{~V}$ for positive $U 56.57$.

In Section 2 we briefly review the results which indicate that the model (3) exhibits $s$-wave superconductivity (or dominant singlet superconducting correlations at large distances in 1D) for certain parameters. At half filling we present evidence of a superconductor-insulator transition. In Section 3 we explain the HF-BCS decoupling scheme and apply it to the electron-hole symmetric case $t_{A B}<t_{A A}=t_{B B}$ at half filling. We obtain a phase diagram separating regions in which the stable phase is a CDW, SDW or triplet $p$-wave superconductor (TS). The latter is the stable one for sufficiently small $U$ and $V$ in a simple cubic lattice in any dimension. In Section 4 we calculate the phase diagram in 1D by a numerical method recently introduced by two of us 40 , which uses topological quantum numbers as order parameters, and compare with the HF-BCS results. Section 5 contains the conclusions.

\section{II. $S$-WAVE SUPERCONDUCTIVITY}

The model (3) becomes simpler when the three-body part of it vanishes. The coefficient of terms of the form $c_{i, \sigma}^{\dagger} c_{j, \sigma} n_{i,-\sigma} n_{j,-\sigma}$ is $t_{3}=2 t_{A B}-t_{A A}-t_{B B}$.

We concentrate first in the results for $t_{3}=0$. Using a HF-BCS decoupling, it has been shown that the model leads to (extended) $s$-wave superconductivity for $V=0$, small enough $U$ and positive (negative) $t^{2}=$ $t_{A A}-t_{A B}$ for a more (less) than half-filled band 41 . In 1D Japaridze and Müller-Hartmann have calculated the correlation exponent $K_{\rho}$ using continuum limit theory and bosonization 3 . They obtained that $K_{\rho}>1$ (superconducting correlations dominate at large distances) if

$$
U<U_{c}=8\left(t_{A B}-t_{A A}\right) \cos (\pi n / 2)-6 V
$$

where $n$ is the number of particles per site.

The particular case $t_{2}=t_{A A}$ corresponds to the relation $t_{A A}=-t_{B}, t_{A B}=0$, and has been exactly solved for $V=027$. Superconducting $\eta$-paired states with off-diagonal long-range order (ODLRO) are part of the highly degenerate ground state. However, as a consequence of this degeneracy, the system does not display anomalous flux quantization (AFQ) for large ring 30 . In other words, the Meissner effect is absent.

Since, due to the rather pathological degeneracy of the ground state, the exact results do not allow to draw definite conclusions for $t_{A B} \neq 0$ while the bosonization results are expected to be valid only for weak interactions, we have carried out a detailed numerical stydy of the model for $V=0$ in rings of 10 and 12 sites 42 . For $t_{A A}=1, t_{A B}=2, t_{B B}=3,2 / 3<n<4 / 5$ and small $U$, 
there are clear indications of binding and AFQ (a tendency towards a periodicity of half a flux quantum in the energy as a function of flux). The calculation of the correlation exponent $K_{\rho}$ indicates that superconducting correlations dominate for $U<U_{c}$, where $U_{c} \sim 9,8$ or 6.5 for $n=0.4,0.5$ or 0.6 electrons per site respectively. These values are nearly 1.4 times larger than the corresponding continuum limit results: $6.46,5.66$ and 4.70 respectively, according to Eq. (44). For $n=0.8, t_{A A}=1$, $t_{A B}=1.5$ and $t_{B B}=2$ we obtain $U_{c}=1.6$, while Eq. (4) gives $U_{c}=1.24$.

The qualitative agreement between Eq. (任) and our numerical results is lost at half filling. While the continuum limit theory predicts that the system is an insulator for all positive values of $U 43$, we find evidence of a superconductor-insulator transition as a function of $U$ when $t_{3}=2 t_{A B}-t_{A A}-t_{B B}=0$ and any sign of $t_{2}=t_{A A}-t_{A B}$ (the sign can be changed using the symmetry properties of the mode 30,42 ). This agrees with previous numerical work of $K_{\rho} 42.44$, a BCS calculation 44 and recent results using slave boson, 45 . For small positive values of $U$ we find in rings of 10 sites that the energy as a function of flux $E(\phi)$ has a form that suggests AFQ for $t_{A A}=1, t_{A B}=0.5$ and $t_{B}=0$. Furthermore, using topological quantum numbers 40 (as explained briefly in Section 4), we detect a transition from charge $\left(\gamma_{c}\right)$ and $\operatorname{spin}\left(\gamma_{s}\right)$ Berry phases $\left(\gamma_{c}, \gamma_{s}\right)=(0,0)$ to $(\pi, \pi)$ (corresponding to the SDW phase) as $U$ increases. We point out that the topological values $(0,0)$ are also obtained for the negative- $U$ Hubbard model which displays singlet $s$ wave superconductivity. The value of $U_{c}$ separating both phases is small. For 10 sites we obtain $U_{c}=0.075$. From the size-dependence we estimate that this actually corresponds to a lower bound. From the results of $K_{\rho}$ of Ref. 22 and using symmetry arguments we estimate (remember $n=1) U_{c}=0.5$ for $t_{A A}=1, t_{A B}=3 / 5$ and $t_{B B}=1 / 5$ and $U_{c}=0.3$ for $t_{A A}=1, t_{A B}=2 / 3$ and $t_{B B}=1 / 3$, but careful finite-size scaling is necessary to give accurate values of $U_{c}$.

While the values of $K_{\rho}$ alone cannot distinguish between singlet $s$-wave (even) and triplet $p$-wave (odd) superconducting states, the BCS results and the Berry phases $(0,0)$ are indicative of the former. A demonstration of the $s$-wave character was provided by the results of stochastic diagonalization by Michielsen and De Raedt 46 which showed the presence of singlet-singlet quasi ODLRO in $1 \mathrm{D}$ for $t_{A A}=1, t_{A B}=1.4$ and $t_{B B}=1.8, n=1.5$ and $U<1$, and also for $t_{A A}=t_{A B}=$ $t_{B B}=1, U=-4$ and $n=1.5$ (negative- $U$ Hubbard model).

We discuss now the effect of the three-body term $t_{3}=2 t_{A B}-t_{A A}-t_{B B}$. When $t_{A A}=t_{B B}$ themodel is electron-hole symmetric for bipartite lattices 42 . For the electron-hole symmetric case with $t_{3}>0$, the meanfield HF-BCS decoupling of the two- and three-body terms leads to singlet $s$-wave (and also $d$-wave in 2D) superconducting solutions 4 . However a positive $t_{3}$ also favours CDW and SDW instabilities 38 . In 2D, the de- tailed (mean-field) calculation of the phase diagram for $V=0$ shows that the SDW is stable for positive $U$ near half filling, while extended $s$-wave superconductivity is present in the ground state for small $U$ or large doping. The $d$-wave paramagnetic solution has always larger energy than the other twot- The electron-hole symmetric case with $t_{3}<0$ is analyzed in the next section.

\section{P-WAVE SUPERCONDUCTIVITY}

In this Section we discus the phase diagram of the model for the electron-hole symmetric case $t_{A B}<t_{A A}=$ $t_{B B}$ and half filling, in simple cubic lattices in arbitrary dimensions using HF-BCS. This decoupling leads naturally to $p$-wave superconductivity for small $U$ and $V$. Although a real proof of the existence of this phase is lacking, further arguments given in the next Section support its existence in $1 \mathrm{D}$. For $t_{A B}=0$, exact results have shown that for small $U$ and $V$, the CDW and SDW phases become unstable because the system lowers its energy taking advantage of the kinetic energy terms $t_{A A}$ and $t_{\text {m }}$ which are inactive in the CDW and SDW phase 26, 55.36 . As mentioned in the Introduction, a phase diagram valid for several lattices in arbitrary dimensions was constryeted 36 . For $t_{A B}=V=0$, an exact solution exists in $1 D_{27}^{27} 3$, but these exact results were unable to identify the nature of the third phpse for finite $t_{A B}$. The numerical and mean-field results3738 show the presence of mobile carriers and a non-vanishing Drude weight within the $\mathrm{M}$ phase in $1 \mathrm{D}$ and $2 \mathrm{D}$, suggesting that the system is a Luttinger liquid in $1 \mathrm{D}$ and metallic in 2D for small $U$ and $V$. However, a suggestion that this phase has dominant triplet superconducting correlations at large distances was given only recently 40 .

Note that if in the three-body part of the correlated hopping (see Eq. 3), the operator $c_{i, \sigma}^{\dagger} c_{j, \sigma}$ is replaced by its expectation value $\tau$ (assumed for this argument independent of spin and nearest-neighbour pair, as in the non-interacting case), this term takes the form $t_{3} \tau \sum_{\langle i j\rangle} n_{i,-\sigma} n_{j,-\sigma}$, with $t_{3}=2 t_{A B}-t_{A A}-t_{B B}<0$. Thus, the three-body term reduces to an attraction of nearest-neighbour electrons with the same spin and triplet pairing is a natural consequence of it.

The HF-BCS decoupling is the most convenient meanfield approximation to reduce the many-body terms of the Hamiltonian to one-body terms. It is a generalization of the prpcedure used by Foglio and Falicov for the normal case20. A more direct way of obtaining the HFBCS Hamiltonian $\left(H_{H F B C S}\right)$ is to define the vacuum as the (unknown for the moment) Slater determinant that is the ground state of $H_{H F B C S}$. Then one should normal order the exact Hamiltapian with respect to this vacuum using Wick's theorem $\$ 5$. Clearly the contractions that appear using this theorem are the HF-BCS expectation values. The exact Hamiltonian takes the form of the HFBCS ground-state energy plus normal ordered one- and 
many-body terms. Neglecting the latter, one obtains the HF-BCS Hamiltonian.

Neglecting also for simplicity spin-flip expectation values of the form $\left\langle c_{i, \sigma}^{\dagger} c_{j,-\sigma}\right\rangle$ (related to spiral spin structures), we obtain $H_{H F B C S}$ using the following approximations for the two- and three-body terms of $H$ :

$$
\begin{aligned}
n_{i \uparrow} n_{i \downarrow} \simeq & \left\langle n_{i \uparrow}\right\rangle n_{i \downarrow}+n_{i \uparrow}\left\langle n_{i \downarrow}\right\rangle-\left\langle n_{i \uparrow}\right\rangle\left\langle n_{i \downarrow}\right\rangle \\
& +\left(c_{i \uparrow}^{\dagger} c_{i \downarrow}^{\dagger}\left\langle c_{i \downarrow} c_{i \uparrow}\right\rangle+h . c .\right)-\left|\left\langle c_{i \downarrow} c_{i \uparrow}\right\rangle\right|^{2} \\
\left(n_{i \uparrow}+n_{i \downarrow}\right)\left(n_{j \uparrow}+n_{j \downarrow}\right) \simeq\left\langle n_{i \uparrow}+n_{i \downarrow}\right\rangle\left(n_{j \uparrow}+n_{j \downarrow}\right) & \\
+ & \left(n_{i \uparrow}+n_{i \downarrow}\right)\left\langle n_{j \uparrow}+n_{j \downarrow}\right\rangle-\left\langle n_{i \uparrow}+n_{i \downarrow}\right\rangle\left\langle n_{j \uparrow}+n_{j \downarrow}\right\rangle \\
+ & \sum_{\sigma}\left\{-\left(\left\langle c_{i \sigma}^{\dagger} c_{j \sigma}\right\rangle c_{j \sigma}^{\dagger} c_{i \sigma}+h . c .\right)+\left|\left\langle c_{i \sigma}^{\dagger} c_{j \sigma}\right\rangle\right|^{2}\right. \\
+ & \left.\sum_{\sigma^{\prime}}\left[\left(\left\langle c_{i \sigma}^{\dagger} c_{j \sigma^{\prime}}^{\dagger}\right\rangle c_{j \sigma^{\prime}} c_{i \sigma}+h . c .\right)-\left|\left\langle c_{i \sigma}^{\dagger} c_{j \sigma^{\prime}}^{\dagger}\right\rangle\right|^{2}\right]\right\},
\end{aligned}
$$

$$
\begin{aligned}
c_{i \uparrow}^{\dagger} c_{j \uparrow}\left(n_{i \downarrow}+n_{j \downarrow}\right) \simeq & \left\langle c_{i \uparrow}^{\dagger} c_{j \uparrow}\right\rangle\left(n_{i \downarrow}+n_{j \downarrow}\right)+c_{i \uparrow}^{\dagger} c_{j \uparrow}\left(\left\langle n_{i \downarrow}\right\rangle+\left\langle n_{j \downarrow}\right\rangle\right. \\
& -\left\langle c_{i \uparrow}^{\dagger} c_{j \uparrow}\right\rangle\left(\left\langle n_{i \downarrow}\right\rangle+\left\langle n_{j \downarrow}\right\rangle\right) \\
& +c_{i \uparrow}^{\dagger} c_{j \downarrow}^{\dagger}\left\langle c_{j \downarrow} c_{j \uparrow}\right\rangle+c_{i \uparrow}^{\dagger} c_{i \downarrow}^{\dagger}\left\langle c_{i \downarrow} c_{j \uparrow}\right\rangle \\
& +\left\langle c_{i \uparrow}^{\dagger} c_{j \downarrow}^{\dagger}\right\rangle c_{j \downarrow} c_{j \uparrow}+\left\langle c_{i \uparrow}^{\dagger} c_{i \downarrow}^{\dagger}\right\rangle c_{i \downarrow} c_{j \uparrow} \\
& -\left\langle c_{i \uparrow}^{\dagger} c_{j \downarrow}^{\dagger}\right\rangle\left\langle c_{j \downarrow} c_{j \uparrow}\right\rangle-\left\langle c_{i \uparrow}^{\dagger} c_{i \downarrow}^{\dagger}\right\rangle\left\langle c_{i \downarrow} c_{j \uparrow}\right\rangle,
\end{aligned}
$$

and the same interchanging spin up and down. Choosing one spin orientation for the sake of clarity, the three-body terms are replaced as:

$$
\begin{aligned}
c_{i \uparrow}^{\dagger} c_{j \uparrow} n_{i \downarrow} n_{j \downarrow}= & c_{i \uparrow}^{\dagger} c_{j \uparrow} c_{i \downarrow}^{\dagger} c_{i \downarrow} c_{j \downarrow}^{\dagger} c_{j \downarrow} \\
\simeq & c_{i \uparrow}^{\dagger} c_{j \uparrow}\left(\left\langle n_{i \downarrow}\right\rangle\left\langle n_{j \downarrow}\right\rangle+\left|\left\langle c_{i \downarrow}^{\dagger} c_{j \downarrow}^{\dagger}\right\rangle\right|^{2}-\left|\left\langle c_{i \downarrow}^{\dagger} c_{j \downarrow}\right\rangle\right|^{2}\right) \\
& -c_{i \downarrow}^{\dagger} c_{j \downarrow}\left(\left\langle c_{i \uparrow}^{\dagger} c_{j \uparrow}\right\rangle\left\langle c_{j \downarrow}^{\dagger} c_{i \downarrow}\right\rangle+\left\langle c_{i \uparrow}^{\dagger} c_{j \downarrow}^{\dagger}\right\rangle\left\langle c_{i \downarrow} c_{j \uparrow}\right\rangle\right) \\
& -c_{j \downarrow}^{\dagger} c_{i \downarrow}\left(\left\langle c_{i \uparrow}^{\dagger} c_{j \uparrow}\right\rangle\left\langle c_{i \downarrow}^{\dagger} c_{j \downarrow}\right\rangle+\left\langle c_{i \uparrow}^{\dagger} c_{i \downarrow}^{\dagger}\right\rangle\left\langle c_{j \downarrow} c_{j \uparrow}\right\rangle\right) \\
& +n_{i \downarrow}\left(\left\langle c_{i \uparrow}^{\dagger} c_{j \uparrow}\right\rangle\left\langle n_{j \downarrow}\right\rangle+\left\langle c_{i \uparrow}^{\dagger} c_{j \downarrow}^{\dagger}\right\rangle\left\langle c_{j \downarrow} c_{j \uparrow}\right\rangle\right) \\
& +n_{j \downarrow}\left(\left\langle c_{i \uparrow}^{\dagger} c_{j \uparrow}\right\rangle\left\langle n_{i \downarrow}\right\rangle+\left\langle c_{i \uparrow}^{\dagger} c_{i \downarrow}^{\dagger}\right\rangle\left\langle c_{i \downarrow} c_{j \uparrow}\right\rangle\right) \\
& +c_{i \uparrow}^{\dagger} c_{i \downarrow}^{\dagger}\left(\left\langle c_{i \downarrow} c_{j \uparrow}\right\rangle\left\langle n_{j \downarrow}\right\rangle-\left\langle c_{j \downarrow} c_{j \uparrow}\right\rangle\left\langle c_{j \downarrow}^{\dagger} c_{i \downarrow}\right\rangle\right) \\
& +c_{i \uparrow}^{\dagger} c_{j \downarrow}^{\dagger}\left(\left\langle n_{i \downarrow}\right\rangle\left\langle c_{j \downarrow} c_{j \uparrow}\right\rangle-\left\langle c_{i \downarrow} c_{j \uparrow}\right\rangle\left\langle c_{i \downarrow}^{\dagger} c_{j \downarrow}\right\rangle\right) \\
& +c_{i \downarrow} c_{j \uparrow}\left(\left\langle c_{i \uparrow}^{\dagger} c_{i \downarrow}^{\dagger}\right\rangle\left\langle n_{j \downarrow}\right\rangle-\left\langle c_{i \uparrow}^{\dagger} c_{j \downarrow}^{\dagger}\right\rangle\left\langle c_{i \downarrow}^{\dagger} c_{j \downarrow}\right\rangle\right) \\
& +c_{j \downarrow} c_{j \uparrow}\left(\left\langle c_{i \uparrow}^{\dagger} c_{j \downarrow}^{\dagger}\right\rangle\left\langle n_{i \downarrow}\right\rangle-\left\langle c_{i \uparrow}^{\dagger} c_{i \downarrow}^{\dagger}\right\rangle\left\langle c_{j \downarrow}^{\dagger} c_{i \downarrow}\right\rangle\right) \\
& +\left(c_{i \downarrow}^{\dagger} c_{j \downarrow}^{\dagger}\left\langle c_{j \downarrow} c_{i \downarrow}\right\rangle+c_{j \downarrow} c_{i \downarrow}\left\langle c_{i \downarrow}^{\dagger} c_{j \downarrow}^{\dagger}\right\rangle\right)\left\langle c_{i \uparrow}^{\dagger} c_{j \uparrow}\right\rangle \\
& +2\left[\left\langle c_{i \uparrow}^{\dagger} c_{j \uparrow}\right\rangle\left(\left|\left\langle c_{i \downarrow}^{\dagger} c_{j \downarrow}\right\rangle\right|^{2}-\left\langle n_{i \downarrow}\right\rangle\left\langle n_{j \downarrow}\right\rangle\right)\right. \\
& +\left\langle c_{i \uparrow}^{\dagger} c_{i \downarrow}^{\dagger}\right\rangle\left(\left\langle c_{j \downarrow} c_{j \uparrow}\right\rangle\left\langle c_{j \downarrow}^{\dagger} c_{i \downarrow}\right\rangle-\left\langle c_{i \downarrow} c_{j \uparrow}\right\rangle\left\langle n_{j \downarrow}\right\rangle\right) \\
& +\left\langle c_{i \uparrow}^{\dagger} c_{j \downarrow}^{\dagger}\right\rangle\left(\left\langle c_{i \downarrow} c_{j \uparrow}\right\rangle\left\langle c_{i \downarrow}^{\dagger} c_{j \downarrow}\right\rangle-\left\langle c_{j \downarrow} c_{j \uparrow}\right\rangle\left\langle n_{i \downarrow}\right\rangle\right) \\
& \left.-\left\langle c_{i \uparrow}^{\dagger} c_{j \uparrow}\right\rangle\left|\left\langle c_{i \downarrow}^{\dagger} c_{j \downarrow}^{\dagger}\right\rangle\right|^{2}\right] .
\end{aligned}
$$

Each thermodynamic phase of the model in the HF-BCS approximation is characterized by a different Destices $A$ and B, in such a way that the nearestneighbours of any site of $\mathrm{A}$ lie in $\mathrm{B}$. In this case, for the SDW $\left\langle n_{i, \sigma}\right\rangle=\left(1+m \sigma e^{i \mathbf{Q} \cdot \mathbf{R}_{\mathbf{i}}}\right) / 2$ while for the CDW

symmetry breaking of the expectation values entering Eqs. (5-8) with respect to the unperturbed system. Since singlet $s$ - and $d$-wave solutions do not exist for $t_{A B}<04$, we have looked for triplet $p$-wave superconductivity (TS). Based on symmetry properties expected for the ground state in 1D (explained at the end of the next section), we assumed that only the $S_{z}= \pm 1$ components of the triplet order parameter do not vanish like in the Anderson-Brinkman-Model phase of superfluid ${ }^{3} \mathrm{He} 60$. For this case we have $\left\langle c_{i \uparrow}^{\dagger} c_{j \downarrow}^{\dagger}\right\rangle$ for all $i, j$ in one lattice direction and a vector $\delta$ connecting nearest-neighbours $\left\langle c_{i+\delta, \sigma}^{\dagger} c_{i, \sigma}^{\dagger}\right\rangle=-\left\langle c_{i-\delta, \sigma}^{\dagger} c_{i, \sigma}^{\dagger}\right\rangle=\psi \neq 0$, while in other directions $\left\langle c_{i+\delta, \sigma}^{\dagger} c_{i, \sigma}^{\dagger}\right\rangle=0$. As a consequence also $\left\langle c_{i+\delta, \sigma}^{\dagger} c_{i, \sigma}\right\rangle$ depends on direction. We also considered the usual CDW and SDW phases for which the cubic lattice in $\mathrm{D}$ dimensions is divided into two equal interpenetrating $\left\langle n_{i, \sigma}\right\rangle=\left(1+\Delta e^{i \mathbf{Q} \cdot \mathbf{R}_{\mathbf{i}}}\right) / 2$ with $\vec{Q}=(\pi, \pi, \ldots, \pi)$ and $m$ and $\Delta$ order parameters. The resulting one-particle Hamiltonian has the form of the non-interacting one with a renormalized effective hopping $t_{e f f}$, plus a symmetry breaking perturbation which, at half filling, depends on two or three parameters to be determined selfconsistently. These parameters are the corresponding order parameter ${ }^{(7)}(\psi, m$ or $\Delta)$ and the different values of $\left\langle c_{i+\delta, \sigma}^{\dagger} c_{i, \sigma}\right\rangle$ : one for CDW, SDW, or TS in 1D, two for TS in more than 1D (for TS in more than 1D $t_{\text {eff }}$ becomes anisotropic).

The phase diagram has a natural energy scale which we call $E$ : the absolute value of the energy of the halffilled non-interacting case for hopping $\left|t_{3}\right|=t_{A A}+t_{B B}-$ $2 t_{A B}$, counting both spins. The ratio $E /\left|t_{3}\right|$ depends only on the dimension of the simple cubic lattice and is $4 / \pi=1.273$ in $1 \mathrm{D} 16 / \pi^{2}=1.621$ in $2 \mathrm{D}, 2.005$ in $3 \mathrm{D} 61$ and 0.798 in $\infty \mathrm{D} 61$. The only point in the $U-V$ phase diagram for which the paramagnetic solution is stable is the triple point $\left(U_{t}, V_{t}\right)=(E, E / z)$ where $z=2 D$ is the coordination number. If and only if $V<V_{t}$, for any $U$, the paramagnetic solution is unstable against the TS phase; if and only if $U>U_{t}=E$, it is unstable against SDW; and if and only if $V>(E+U) / 2 z$ it is unstable against the CDW. These boundaries allow us to establish that, in any dimension, inside the region bounded by the dashed line in Fig. 1, the TS is the stable phase. Also the CDW-TS boundary lies between the dashed and dot-dashed lines of Fig. 1. With an adequate change of variables, it can be shown that the self-consistency problems for the CDW and SDW take the same form on the line $U=z V$. Thus, in any dimension, for $U \geq E$, $U=z V$ is the boundary of the CDW-SDW first-order transition, independently of the other parameters of the model.

The above results are quite general and valid in any dimension. The region of stability of the TS extends always beyond the dashed lines of Fig. 1 and the precise location of the boundaries of the TS depends on $t_{A B}$ and 
the dimension. In Fig. 1, the specific case of $1 \mathrm{D}$ and $t_{A A}=t_{B B}=1, t_{A B}=0.2$ is shown. The change in slope of the SDW-TS boundary is due to a metamagnetic firstorder transition inside the SDW from large to small order parameter as $V$ increases. A similar situation occurs in the CDW-TS boundary. Near the triple point, and particularly for $t_{A B} \sim 1$, all order parameters become very small, and the numerical method used to solve the selfconsistency equations breaks down. Similarly, in 1D, for $V=0$ and $t_{A B}=0.097$, the effective hopping vanishes in the paramagnetic phase and the HF-BCS approximation becomes invalid for $t_{A B} \sim 0.1$ or smaller.

One expects that the HF-BCS results are reliable for small values of the interactions (small $U, V$ and $1-t_{A B}$, with $t_{A A}=t_{B B}=1$ ) and $\mathrm{D}>1$, supporting the existence of the $p$-wave TS for these parameters. In $1 \mathrm{D}$ there is no true LRO and the HF-BCS results only have qualitative validity. A comparison with more accurate methods in $1 \mathrm{D}$ is made in the next section.

\section{PHASE DIAGRAM OBTAINED FROM TOPOLOGICAL TRANSITIONS}

In recent years, the concept of Berry phase was a subject of great interest in a variety of fields in physics. Zak has shown that it can be used for labeling energy band in solids 62, and subsequent work showed that changes of polarization are proportional to the corresponding change in a Berry phase 63. Ortiz and Martin have generalized these concepts to a many-body ground state64, and Resta and Sorella used this concept to identify a ferroelectric transition 65. This many-body Berry phase is simply the phase captured by the ground state in a ring of $L$ sites as the boundary conditions $c_{i+L, \sigma}^{\dagger} \overline{\bar{\sigma}} e^{i \phi} c_{j, \sigma}^{\dagger}$, complete a cycle from a flux $\phi=0$ to $\phi=2 \pi 40,6466$. We call this phase the charge Berry phase $\gamma_{c}$.

While previous work assumed always that the ground state is non-degenerate for all $\phi$ (except at isolated points of the parameter space at which $\gamma_{c}$ is indefinite), two of us have recently generalized this concept for the case in which there is a-crossing of levels in the ground state as a function of $\phi$. This is the case of the superconducting phases, for which there is $\mathrm{AFQ}$ as a consequence of the crossing energy levels. Furthermore, a "spin" Berry phase $\gamma_{s}$ was defined as that captured by the ground state in the cycle $0 \leq \phi \leq 2 \pi$ varying the boundary conditions as $c_{i+L, \sigma}^{\dagger}=e^{i \sigma \phi} c_{i, \sigma}^{\dagger}$ with $\sigma=1$ (-1) for spin up (down) 40 . Due to the inversion symmetry of the model Eq. (3). the Berry phases are quantized and in $1 \mathrm{D}$ can take only the values 0 and $\pi$ (modulo $2 \pi$ ). Thus, it turns out that $\gamma_{c} / \pi$ and $\gamma_{s} / \pi$ are topological quantum numbers which are related to changes in the total polarization and the difference between polarizations for spin up and down respectively 40 .

Defining a vector $\gamma=\left(\gamma_{c}, \gamma_{s}\right)$, it is easy to see 40 that for a CDW (SDW) with maximum order parameter one has $\gamma=(0,0)(\gamma=(\pi, \pi))$, and these values are consistent with the change in up and down polarizations when the electrons of spin down of one phase are moved to the other sublattice to form the other phase. Since $\gamma$ jumps in $\pi$ only at the phase transitions, the values $\gamma(\mathrm{CDW})=(0,0)$ and $\gamma(\mathrm{SDW})=(\pi, \pi)$ are valid for any non-zero magnitude of the corresponding order parameters. These values are also consistent with the canonical transformation.

$$
c_{j, \uparrow}^{\prime}=c_{j, \uparrow}, c_{j, \downarrow}^{\prime}=(-1)^{j} c_{j, \downarrow}^{\dagger}
$$

under which the CDW and SDW are interchanged and the Berry phases modulo $\pi$ transform as:

$$
\gamma^{\prime}=\gamma+\pi
$$

Since the ground state for $U=V=0$ is invariant under Eq. (9), Eq. (10) implies that for the third phase present in the diagram $\gamma_{c}(T S)=\gamma_{s}(T S)+\pi$ (numerically it turns out that $\gamma(T S)=(0, \pi))$, and thus, at least one of the topological numbers $\gamma_{c} / \pi, \gamma_{s} / \pi$ jumps at each boundary. Thus, this method combined with finite-size scaling is able to determine accurately the phase diagram (see Fig. 2).

The best previous numerical methods to determine phase diagrams of this type were based on the size dependence of SDW and CDW order parameters, and their probability densities in a Monte Carlo sampling 33 ,52.54. However, these quantities as well as different correlation functions vary smoothly at the transitipp and it is very difficult to obtain accurate boundaries 5255 . Instead, the use of topological numbers as order parameters necessarily leads to sharp transitions.

In Fig. 2 we show the phase diagram of the model Eq. (3) in 1D obtained with the above mentioned method, for two values of $t_{A B}$, and compare it with the corresponding $\mathrm{HF}$ BCS results and with the exact result for $t_{A B} \rightarrow 036$. Although, as mentioned in the previous section, the HF BCS results are not expected to have quantitative validity in $1 \mathrm{D}$ for large values of the interaction, for small $t_{A B}$ the resulting phase diagram is in reasonable agreement with that obtained with the above explained more reliable numerical method. The results of the latter tend to the exact phase diagram as $t_{A B} \rightarrow 0$. The comparison also shows that the TS phase in $1 \mathrm{D}$ extends beyond the expectations of the HF BCS results. This is particularly clear for $t_{A B} \sim t_{A A}=t_{B B}$ and $U \sim 2 \mathrm{~V}$, and is probably related to the fact that, in the continuum limit theory, the backscattering and Umklapp terms coming from the $U$ and $V$ terms of the Hamiltonian (ultimately responsible of the insulating hehaviour), nearly cancel each other on the line $U=2 V 54,56$.

We should note that the method of the topological transitions alone, is not able to identify the nature of the TS phase, since different phases can have the same topological numbers. For example, we find $\gamma=(0,0)$ not only for the CDW, but also in the negative- $U$ Hubbard model with small negative $V$, for which $s$-wave singlet pairing 
occurs 565. We also find it for our model (Eq. (3)) with $t_{3}=V=0$ and small $U$, for which also singlet superconducting correlations dominate at large distances, as explained in Section 2.

In the following we summarize the evidence in favour of the triplet superconducting phase in 1D.

a) Superconductivity:

- Since each time the Berry phase is evaluated, the ground state energy as a function of the flux $E(\phi)$ is computed at the same time, we have checked that there is a tendency to AFQ (i.e. $E(\phi) \simeq E(\phi+\pi)$ ) inside almost all the TS phase.

- Our previous calculations of the correlation exponent $K_{\rho}$ for $V=0$ show the dominance of superconducting correlations at large distances $\left(K_{\rho}>1\right)$ for $U<J$, and an opening of a charge gap for $U>U_{c} 3942$. The value of $U_{c}$ has been estimated as $U_{c} \sim 3.5$ for $t_{A B}=0.2$ and $t_{A A}=t_{B B}=142$ in good agreement with the result shown in Fig. 2. For $t_{A B}=0.6$, from $K_{\rho}$ one obtains $U_{c}=2.05 \pm 0.0539$, while from the topological transition we obtain $U_{c}=2.1140$.

- For a "metallic" gapless phase with $K_{\rho} \leq 1$ (as in the non-interacting case), one expects undetermined Berry phases 40,66 instead of the result $\gamma=(0, \pi)$ for the TS phase.

b) Triplet character:

- The HF BCS decoupling in any dimension leads to unstable singlet and stable triplet superconductivity.

- For known cases of singlet superconductivity (mentioned above) we obtain $\gamma=(0,0)$ in contrast to the result $\gamma(T S)=(0, \pi)$.

- We find that in rings of 10 sites the nearestneighbour triplet-triplet correlations are larger than the singlet-singlet ones at the largest distance in the ring ( 5 sites).

- Numerically, we obtain a non-degenerate ground state inside the TS phase which should transform into itself under the symmetry transformation (9) for $U=V=0$, since the Hamiltonian is invariant at that point. An ordinary BCS singlet solution transforms into a SDW under this transformation, leading necessarily to a degenerate ground state for $U=V=0$. Instead, using $c_{k, \downarrow}^{\prime \dagger}=c_{-k+Q, \downarrow},|0\rangle=$ $\prod_{k} c_{k, \downarrow}^{\prime \dagger}$ and $\cos \left(k_{\alpha}+Q_{\alpha}\right)=-\cos \left(k_{\alpha}\right)$, it can be easily checked that our $p$-wave triplet BCS solution in the $x$-direction (see Section 3 after Eq. (8)):

$$
\left.\left|p_{x}>=\prod_{k \sigma, k_{x}>0}\left(u_{k}+v_{k} c_{k, \sigma}^{\dagger} c_{-k, \sigma}^{\dagger}\right)\right| 0\right\rangle
$$

is invariant under Eq. (9).
- Finite-size scaling is consistent with the absence of a spin gap inside the TS phase. Continuum-limit theory predicts that triplet superconducting correlations dominate at large distances (due to logarithmic corrections) when $K_{\rho}>1$ and a spin gap is absent 67 .

\section{SUMMARY AND DISCUSSION}

We have studied the occurrence of superconductivity in the generalized Hubbard model Eq. (3). This model contains the most important physical ingredients expected to describe transition metals in general26 or cuprate superconductors21 24. In $1 \mathrm{D}$, most of the parameters of the model appropriate for systems like transpolyacetylene have been estimated 25,4 and are consistent with several regions of parameters for which we find superconductivity.

When the three-body term $t_{3}=2 t_{A B}-t_{A A}-t_{B B}$ vanishes, singlet $s$-wave superconductivity is expected for electron densities per site $n \neq 1$ or very small values of $U$. For $n$ not too near one and $V=0$, Eq. (4) describes qualitatively the values of $U$ below which superconducting correlations dominate at large distances in 1D. However, numerical results suggest that Eq. (4) underestimates these values by a factor $\sim 1.4$. For $n=1$, the region of superconducting behaviour is very small.

Instead, for $n=1$ and $t_{A B}<t_{A A}=t_{B B}=1$, we find evidence of a $p$-wave triplet superconducting phase (TS) for small values of $U$ and $V$. The Hartree-Fock BCS approximation gives a stable TS for sufficiently small $U$ and $V$ in any dimension. In 1D, the numerical method of the Berry phases, described in the previous section, predicts a stable TS even for small $1-t_{A B}$ and large values of $U \sim 3$ near the line $U=2 \mathrm{~V}$.

While a definite proof of the triplet character of this phase does not exist so far, there are several arguments in favour if it enumerated at the end of the previous section. In contrast to the previous case of $s$-wave superconductivity $\left(2 t_{A B}=t_{A A}+t_{B B}\right)$ mentioned above, for $t_{A B}<t_{A A}=t_{B B}$, the effect of doping seems to weaken the TS in favour of the SDW at $V=042$.

Experimentally, evidence of a triplet odd-parity superconducting order parameter exists in some quasi$1 \mathrm{D}$ organic conductors 68 . In $1 \mathrm{D}$, our solution is compatible with a nodeless superconducting gap, as evidenced by thermal conductivity measurements in $(\mathrm{TMTSF})_{2} \mathrm{ClO}_{4} 68$.

The effect of phonons, particularly in 1D, can stabilize insulating CDW states, or favour $s$-wave superconducting states. However, based on the general study performed in 1D using bosonization 67, we believe that our results remain qualitatively valid in presence of a small or moderate electron-phonon coupling. In addition, if phonons are treated in the antiadiabatic approximation, the model Eq. (3) retains its form [21b]. 


\section{ACKNOWLEDGMENTS}

Two of us (A. A. A. and E. G.) would like to thank Prof. J. Voit for useful discussions concerning the nature of the $p$-wave superconducting phase and Prof. G. Ortiz for discussions about topological quantum numbers. E. G. and K. H. are supported by Consejo Nacional de Investigaciones Científicas y Técnicas (CONICET). A. A. A. is partially supported by CONICET.

${ }^{1}$ H. Lin, J. Hirsch and D. Scalapino, Phys. Rev. B 37, (1988) 7359.

${ }^{2}$ M. Imada and Y. Hatsugai, J. Phys. Soc. Jpn. 58, (1989) 3752 .

${ }^{3}$ A. Moreo, Phys. Rev. B 45, (1992) 5059.

${ }^{4}$ E. Dagotto, Rev. Mod. Phys. 66, (1994) 763.

${ }^{5}$ S. Zhang, J. Carlson and J. Gubernatis, Phys. Rev. Lett. 78, (1997) 4486.

${ }^{6}$ E. Dagotto and J. Riera, Phys. Rev. Lett. 70, (1993) 682.

${ }^{7}$ E. Heeb and T. Rice, Europhys. Lett. 27, (1994) 673.

${ }^{8}$ C. Batista, L. Manuel, H. Ceccatto and A. Aligia, Europhys. Lett. 38, (1997) 147 and references therein.

9 M. Ogata, M. Lucchini, S. Sorella and F. Assaad, Phys. Rev. Lett. 66, (1991) 2388.

${ }^{10}$ F. Lema, C. Batista and A. Aligia, Physica C 259, (1996) 287.

${ }^{11}$ E. Lieb and F. Wu, Phys. Rev. Lett. 20, (1968) 1445.

12 P. Schlottmann, Phys. Rev. B 36, (1987) 5177; P. Bares and G. Blatter, Phys. Rev. Lett. 64, (1990) 2567; B. Sutherland, Phys. Rev. B 12, (1975) 3795.

${ }^{13}$ K. Hallberg and C. Balseiro, Solid State Communications, 82, (1992) 523.

14 J. Hirsch, Phys. Lett. A 134, (1989) 452; Physica C 158, (1989) 326.

${ }^{15}$ R. Bariev, J. Phys. A 24, (1991) L549.

${ }^{16}$ R. Bariev, A. Klümper, A. Schadschneider and J. Zittartz, J. Phys. A 26, (1993) 1249; ibid. J. Phys. A 26, (1993) 4863.

${ }^{17}$ M. Quaisser, A. Schadschneider and J. Zittartz, Z. Phys. B 95, (1994) 427.

18 A. Schadschneider, G. Su and J. Zittartz, Z. Phys. B 102, (1997) 397.

19 M. Quaisser, A. Schadschneider and J. Zittartz, Europhys. Lett., 32, (1995) 179.

${ }^{20}$ M. Foglio and L. Falicov, Phys. Rev. B 20, (1979) 4554.

${ }^{21}$ F. Marsiglio and J. Hirsch, Phys. Rev. B 41, (1990) 6435; Phys. Rev. 49, (1994) 1366.

22 M. Simon, M. Baliña and A. Aligia, Physica C 206, (1993) 297; M. Simon and A. Aligia, Phys. Rev. B 48, (1993) 7471.

${ }^{23}$ H. Schüttler and A. Fedro, Phys. Rev. B 45, (1992) 7588.

${ }^{24}$ M. Simon, A. Aligia and E. Gagliano, Phys. Rev. B 56, (1997) 5637 and references therein.

${ }^{25}$ D. Campbell, J. Gammel and E. Loh Jr., Phys. Rev. B 42, (1990) 475 and references therein.
${ }^{26}$ R. Strack and D. Vollhardt, Phys. Rev. Lett 70, (1993) 2673.

${ }^{27}$ L. Arrachea and A. Aligia, Phys. Rev. Lett. 73, (1994) 2240.

${ }^{28}$ J. de Boer, V. Korepin and A. Schadschneider, Phys. Rev. Lett. 74, (1995) 789.

${ }^{29}$ A. Schadschneider, Phys. Rev. B 51, (1995) 10386.

${ }^{30}$ L. Arrachea, A. Aligia and E. Gagliano, Phys. Rev. Lett. 76, (1996) 4396.

${ }^{31}$ I. Karnaukov, Phys. Rev. Lett. 73, (1994) 1130; Int. J. Mod. Phys. B 10, (1996) 3673 and references therein.

${ }^{32}$ G. Bedürftig and H. Frahm, Phys. Rev. Lett. 74, (1995) 5284 .

${ }^{33}$ A. Bracken, M. Gould, J. Links and Y. Zhang, Phys. Rev. Lett. 74, (1995) 2768.

${ }^{34}$ R. Bariev, A. Klümper and J. Zittartz, Europhys. Lett. 32, (1995) 85.

35 A. Ovchinnikov, Mod. Phys. Lett. B 7, (1993) 1397.

36 A. Aligia, L. Arrachea and E. Gagliano, Phys. Rev. B 51, (1995) 13744.

37 E. Gagliano, A. Aligia, L. Arrachea and M. Avignon, Phys. Rev. B 51, (1995) 14012; Physica B 224, (1996) 605.

${ }^{38}$ L. Arrachea, E. Gagliano and A. Aligia, Phys. Rev. B 55, (1997) 1173.

39 L. Arrachea, A. Aligia and E. Gagliano, Physica C 268 , (1996) 233.

40 A. Aligia and E. Gagliano, cond-mat/9712190.

${ }^{41}$ F. Marsiglio and J. Hirsch, Physica C 165, (1990) 71.

${ }^{42}$ L. Arrachea, A. Aligia, E. Gagliano, K. Hallberg and C. Balseiro, Phys. Rev. B 50, (1994) 16044.

${ }^{43}$ G. Japaridze and E. Müller-Hartmann, Ann. Phys. (Leipzig) 3, (1994) 163.

44 M. Airoldi and A. Parola, Phys. Rev. B 51, (1995) 16327.

${ }^{45}$ B. Bulka, Phys. Rev. B (to be published).

${ }^{46}$ K. Michielsen and H. de Raedt, Int. J. Mod. Phys. B 11, (1997) 1311.

${ }^{47}$ L. Arrachea and A. Aligia, Physica C 289, (1997) 70.

48 A. Painelli and A. Girlando, Phys. Rev. B 39, (1989) 2830.

49 A. Fortunelli and A. Painelli, Chem. Phys. Lett. 214, (1993) 402.

${ }^{50}$ F. Buzatu, Phys. Rev. B 49, (1994) 10176.

${ }^{51}$ J. de Boer and A. Schadschneider, Phys. Rev. Lett. 75, (1995) 4298.

52 J. Hirsch, Phys. Rev. Lett. 53, (1984) 2327.

53 J. Cannon and E. Fradkin, Phys. Rev. B 41, (1990) 9435.

54 J. Cannon, R. Scalettar and E. Fradkin, Phys. Rev. B 44, (1991) 5995.

55 B. Fourcade and G. Spronken, Phys. Rev. B 29, (1984) 5089; ibid. 29, (1984) 5096.

56 J. Voit, Phys. Rev. B 45, (1992) 4027.

${ }^{57}$ H. Lin, E. Gagliano, D. Campbell, E. Fradkin and J. Gubernatis, unpublished.

58 J. Blaizot and G. Ripka, Quantum theory of finite systems (MIT, Cambridge, Massachusetts,1986).

59 J. Lorenzana, M. Grynberg, L. Yu K. Yonemitsu and A. Bishop, Phys. Rev. B 47, (1993) 13156.

60 See for example A. Leggett, Rev. Mod. Phys. 47, (1975) 331; V. Mineev, Sov. Phys. Usp. 26, (1983) 160.

${ }^{61}$ H. Yokohama and H. Shiba, J. Phys. Soc. Jpn. 56, (1987) 1490; ibid. 56, (1987) 582. 
62 J. Zak, Phys. Rev. Lett. 62, (1989) 2747; L. Michel and J. Zak, Europhys. Lett. 18, (1992) 239.

${ }^{63}$ R. Resta, Rev. Mod. Phys. 66, (1994) 899.

${ }^{64}$ G. Ortiz and R. Martin, Phys. Rev. B 49, (1994) 14202.

${ }^{65}$ R. Resta and S. Sorella, Phys. Rev. Lett. 74, (1995) 4738.

${ }^{66}$ G. Ortiz, P. Ordejón, R. Martin and G. Chiappe, Phys. Rev. B 54, (1996) 13515.

67 J. Voit, Rep. Prog. in Phys. 58, (1995) 977.

${ }^{68}$ S. Belin and K. Behnia, Phys. Rev. Lett. 79, (1997) 2125, and references therein.
Figure 1: Mean-field (HF BCS) phase diagram of the model (3) for one particle per site and $t_{A A}=t_{B B}>t_{A B}$. The full line is the CDW-SDW boundary in any dimension. Inside the region bounded by the dashed lines, the triplet superconducting (TS) phase is the stable one. The CDW-TS boundary lies between the dashed and dot-dashed lines. $E / 2\left(t_{A A}-t_{A B}\right)$ is a number of order one which depends on dimension (see text). Solid circles (squares) are points on the CDW-TS (SDW-TS) boundary in 1D for $t_{A A}=t_{B B}=1$ and $t_{A B}=0.2$.

Figure 2: Phase diagram of the model (B) in 1D for one particle per site, $t_{A A}=t_{B B}=1$ and two different values of $t_{A B}$. Solid (open) circles are determined from the jump of $\gamma_{c}\left(\gamma_{s}\right)$. Solid squares, dotted and dot-dashed lines are HF BCS results. The dashed lines are exact results in the limit $t_{A B} \rightarrow 036$. Full lines are guides to the eye. The small difference between the results from both Berry phases at the CDW-SDW boundary is due to finite-size effects. 


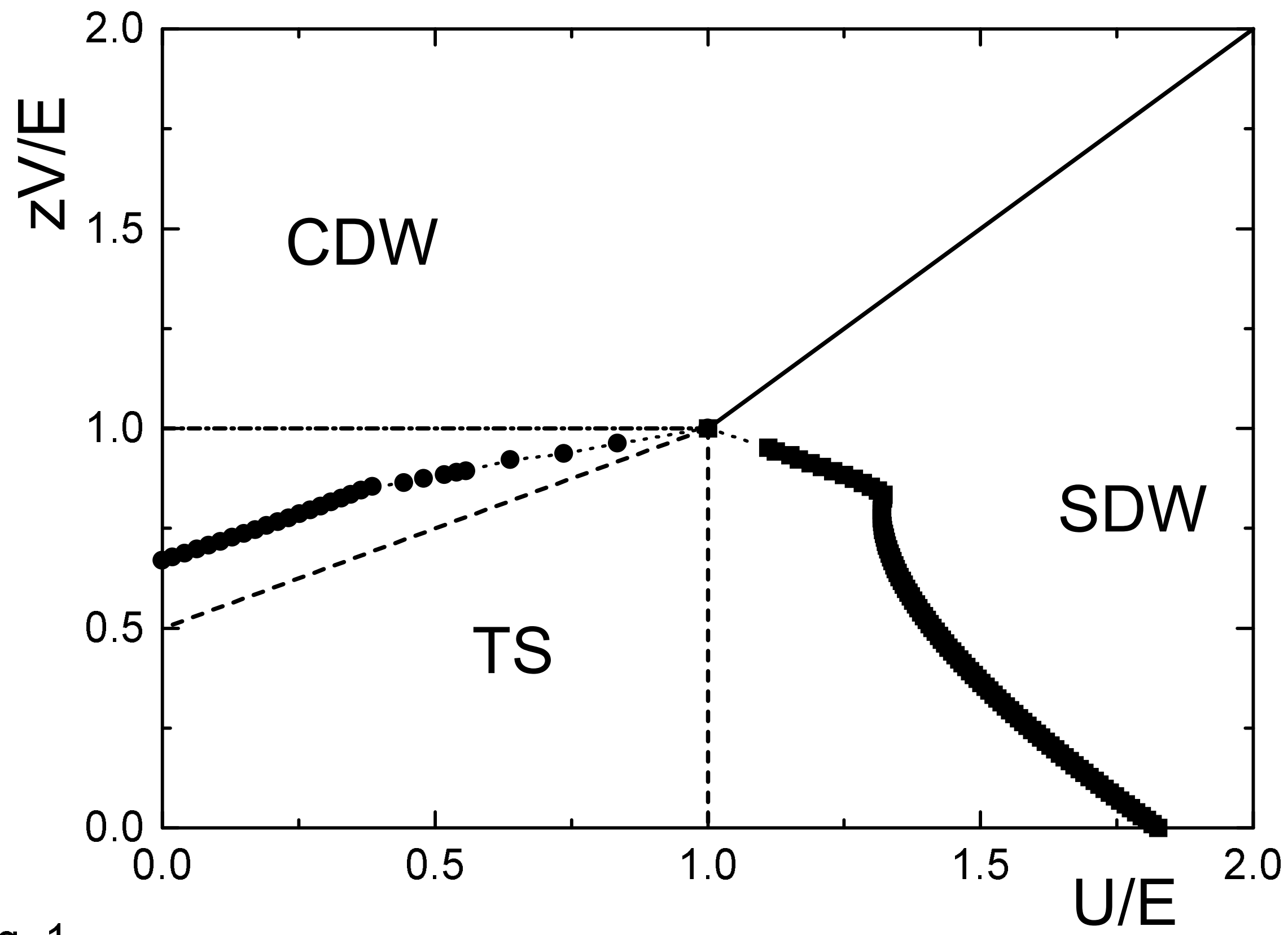

Fig. 1 




Fig. 2 